\title{
Conhecimento dos Médicos Clínicos do Brasil sobre as Estratégias de Prevenção e Tratamento da Osteoporose
}

\section{Brazilian General Practitioner's Knowledge on Osteoporosis Prevention and Treatment Strategies}

\author{
Vera Lúcia Szejnfeld ${ }^{(1)}$, Fagner Jennings ${ }^{(2)}$, Charlles Heldan de Moura Castro ${ }^{(3)}$, \\ Marcelo de Medeiros Pinheiro ${ }^{(4)}$, Antonio Carlos Lopes ${ }^{(5)}$
}

\section{RESUMO}

Objetivos: investigar o conhecimento de profissionais médicos brasileiros a respeito da osteoporose, estratégias de prevenção e tratamento e o acesso à informação em osteoporose, utilizando-se um questionário desenvolvido para avaliar o conhecimento de profissionais médicos em relação à importância da osteoporose e suas estratégias de tratamento. Pacientes e métodos: o questionário foi divulgado a 11 mil médicos afiliados à Sociedade Brasileira de Clínica Médica (SBCM). Realizou-se a divulgação da pesquisa e do questionário por meio de anúncios publicados no Jornal do Clínico. Inicialmente, foi disponibilizado na home-page e, em seguida, diretamente enviado via e-mail aos sócios cadastrados da SBCM. Resultados: um total de 329 questionários retornou aos pesquisadores. A maioria dos médicos $(55,3 \%)$ tinha tempo de graduação superior a 10 anos. Pouco mais que a metade dos profissionais médicos pesquisados $(55 \%)$ relatou ter fácil acesso à densitometria óssea. A maioria (99\%) dos participantes acredita que é importante ou muito importante prevenir a osteoporose. Da mesma forma, $73 \%$ dos médicos pesquisados acreditam que a osteoporose possa ser prevenida e $63 \%$ deles discutem o assunto com os seus pacientes. Por outro lado, mais da metade dos médicos pesquisados não crê que seus pacientes venham a mudar hábitos de vida e cerca de $50 \%$ deles não acreditam que seus pacientes venham a aderir ao tratamento da doença em longo prazo. Apenas 35\% dos médicos pesquisados acreditam que os tratamentos para osteoporose sejam efetivos. Cerca de $82 \%$ fazem uso da densitometria óssea. Médicos com 10 anos ou mais de graduação utilizam a densitometria óssea mais freqüentemente que seus colegas com menos tempo de graduação. Conclusão: os autores acreditam que as informações obtidas no presente estudo poderiam ser úteis para o início do desenvolvimento de estratégias educacionais efetivas para a prevenção e o tratamento de pacientes com osteoporose.

Palavras-chave: conhecimento, médicos generalistas, osteoporose, diagnóstico, tratamento.

\begin{abstract}
Objectives: Brazilian physicians' knowledge on osteoporosis prevention and treatment strategies was investigated in this cross-sectional study. A specific structured questionnaire was designed to evaluate physicians' knowledge regarding osteoporosis prevention and treatment as well as osteoporosis information access. Patients and methods: the questionnaire was made available to around 11,000 physicians affiliated to the Brazilian Society of Internal Medicine (BSIM). The questionnaire was initially presented at the BSIM home-page and subsequently sent by e-mail to all BSIM members. Results: a total of 329 answered questionnaires returned to the researchers. Most of the physicians that answered the questionnaire (55.3\%) had time since graduation lougher than 10 years and half of them (55\%) reported having easy access to bone densitometry. The great majority of the physicians (99\%) believe that preventing osteoporosis is important or very important. Accordingly, 73\% of the physicians believe that osteoporosis can be prevented and around $63 \%$ of them discuss the issue with their patients regularly. On the other hand, most of the physicians that answered the questionnaire do not believe that their patients are able to change life habits or will adhere to the treatment in the longterm. Only 35\% of the physicians believe that current osteoporosis treatment is effective. Around $82 \%$ of the physicians make use of bone densitometry to evaluate osteoporosis. Physicians with time since graduation higher than 10 years reported using bone densitometry more often than their colleagues with less time since graduation. Conclusion: our results demonstrate that educational programs aiming at the general practitioner are needed in order to provide better care in terms of prevention and treatment of skeletal fragility syndromes.
\end{abstract}

Keywords: knowledge, general practitioner, osteoporosis, diagnosis, treatment.

Recebido em 08/05/07. Aprovado, após revisão, em 01/07/07. Declaramos a inexistência de conflitos de interesse.

Estudo referente à Disciplina de Reumatologia da EPM/Unifesp e financiado pela Fapesp, processo 03/09402-2, iniciação científica

1. Professora adjunta da Disciplina de Reumatologia do Departamento de Medicina da Escola Paulista de Medicina da Universidade Federal de São Paulo (EPM/Unifesp).

2. Graduando em Medicina da EPM/Unifesp.

3. Professor visitante da Disciplina de Reumatologia do Departamento de Medicina da EPM/Unifesp

4. Médico-assistente da Disciplina de Reumatologia do Departamento de Medicina da EPM/Unifesp.

5. Professor titular da Disciplina de Clínica Médica do Departamento de Medicina da EPM/Unifesp.

Endereço para correspondência: Vera Lúcia Szejnfeld, Rua Eliseu Visconti, 200, CEP 05683-010, São Paulo, SP, Brasil, e-mail: vera@cura.com.br 


\section{INTRODUÇÃO}

A osteoporose é um problema de saúde pública mundial cuja importância e cujo impacto vêm crescendo nas últimas décadas. Graças aos avanços da medicina, as pessoas estão atingindo idades mais avançadas e, desta forma, a maioria das populações está envelhecendo. Uma pesquisa realizada na Comunidade Européia em meados da década de 1990 estima que até o ano 2040 o número de idosos irá dobrar nesse continente e, em conseqüência, o número de fraturas devidas à osteoporose irá aumentar entre $50 \%$ e $300 \%{ }^{(1)}$.

A osteoporose provoca efeitos devastadores associados ao elevado impacto socioeconômico na população geral ${ }^{(2)}$. $\mathrm{Na}$ Espanha, estima-se que três milhões de mulheres e 750 mil homens tenham osteoporose ${ }^{(3)}$. No Canadá, a doença afeta aproximadamente 1,4 milhão de indivíduos, principalmente mulheres na pós-menopausa e idosas ${ }^{(4)}$.

Vários estudos têm sido realizados com o intuito de determinar o custo do tratamento das fraturas causadas pela osteoporose. Na Inglaterra, foram gastos 942 milhões de libras no ano de 1998 com o tratamento das fraturas associadas à osteoporose ${ }^{(5)}$. Além do elevado custo social e econômico, as fraturas associadas à osteoporose apresentam consideráveis mortalidade e morbidade. Indivíduos com fraturas de quadril apresentam taxa de mortalidade de $30 \% \mathrm{em} 1$ ano. Indivíduos com idade superior a 70 anos são particularmente de alto risco e estão entre os pacientes com maior mortalidade. Cerca de $46 \%$ dos pacientes com fraturas de quadril desenvolverão dependência de cuidados de terceiros para as atividades da vida diária e boa parte deles estará confinada em suas residências 1 ano após a fratura ${ }^{(6)}$. O aumento da expectativa de vida da população mundial tem levado a projeções que estimam o triplo do número de fraturas de quadril em 2050 em comparação com o início da década de $1990^{(7)}$.

Embora a baixa massa óssea e o risco de fragilidade esquelética possam ser identificados pela densitometria óssea e o tratamento específico da osteoporose reduza em $50 \%$ o risco de fratura, o rastreamento populacional com exames de densitometria e/ou ultra-sonometria óssea parece não ser factível. Especialmente em países pobres ou em desenvolvimento, onde há restrição crônica de recursos disponíveis para estratégias de promoção da saúde, o rastreamento da osteoporose apresenta custos proibitivos. A avaliação clínica dos fatores de risco para identificar pacientes que apresentam maior risco de osteoporose e fraturas parece ser a melhor forma de seleção e uma estratégia eficaz para minimizar o impacto da doença ${ }^{(8,9)}$.
Várias outras estratégias têm sido propostas com o propósito de reduzir o impacto da osteoporose e incluem mecanismos para otimizar o diagnóstico e o tratamento da doença. Estratégias de conscientização populacional a respeito da doença e suas implicações parecem ser promissoras. Outro enfoque importante é a conscientização dos profissionais de saúde que lidam com indivíduos potencialmente em risco.

Uma pesquisa recente da Sociedade Nacional de Osteoporose (NOS) ${ }^{(10)}$, de Londres, avaliou o conhecimento dos clínicos gerais em atividade na Inglaterra a respeito do diagnóstico e do tratamento da osteoporose. O estudo demonstrou que os clínicos gerais preocupam-se muito com o impacto da osteoporose sobre a saúde pública. No entanto, na prática clínica diária, poucos estavam envolvidos no tratamento de indivíduos com osteoporose. Outro estudo também conduzido entre ingleses revelou que dois terços dos clínicos gerais ainda não estavam convencidos a respeito da eficácia do tratamento medicamentoso e que a educação sobre osteoporose era inadequada ${ }^{(1)}$.

Neste estudo, avaliamos o conhecimento de profissionais médicos brasileiros, clínicos gerais, a respeito da osteoporose, estratégias de prevenção e tratamento, bem como sobre o acesso à informação em osteoporose.

\section{CASUÍSTICA E MÉTODO}

Um questionário originalmente desenhado para avaliar o conhecimento de profissionais médicos em relação à importância da osteoporose e suas estratégias de tratamento nos Estados Unidos foi traduzido e adaptado para ser empregado na presente pesquisa.

O questionário estruturado aborda informações sobre epidemiologia, impacto da osteoporose, freqüência de abordagem do tema osteoporose na graduação e pós-graduação médica e o conhecimento do médico sobre diagnóstico e tratamento da osteoporose, além de questionamentos sobre a existência de serviços especializados. Consta de 27 questões com um total de 66 itens individuais de informação, incluindo espaço aberto para comentários.

Disponibilizou-se o questionário a 11 mil sócios profissionais médicos afiliados à Sociedade Brasileira de Clínica Médica (SBCM) por meio de sua webpage. Realizou-se a divulgação da pesquisa e do questionário por meio de anúncios publicados no Jornal do Clínico, nas edições de julho/agosto de 2003 e 2004.

A pesquisa foi realizada em duas etapas. Na primeira fase, disponibilizou-se o questionário na home-page da 
SBCM entre os meses de julho de 2003 e agosto de 2004 . A segunda fase da pesquisa compreendeu os meses de setembro a dezembro de 2004 , período em que o questionário foi diretamente enviado via e-mail aos sócios cadastrados na SBCM. Com o questionário, vinha uma observação alertando aos médicos que já houvessem respondido ao questionário não o fizessem uma segunda vez.

Dados demográficos dos participantes, bem como o tempo de graduação, foram coletados e analisados. Os dados obtidos com a aplicação do questionário foram tabulados e expressos em percentagem.

Utilizando o Teste do Qui-Quadrado, avaliaram-se potenciais associações entre o tempo de graduação e as diversas respostas dadas a questões específicas do questionário.

\section{RESULTADOS}

Dos 11 mil questionários disponibilizados na home-page da SBCM, apenas 329, ou seja, 3\% dos médicos responderam ao questionário. Conforme se demonstrou na Tabela 1 , a maioria dos participantes era do gênero masculino (61\%) e tinha tempo de graduação superior a 10 anos. A maioria dos médicos que preencheram o questionário era das Regiões Sudeste e Sul, 74\% e 11\%, respectivamente. Cerca de $59 \%$ deles residiam e trabalhavam em cidades do interior, enquanto $42 \%$ eram procedentes das capitais do Brasil.

TABELA 1

Profissionais médicos afiliados à Sociedade Brasileira de Clínica Médica que responderam ao Questionário SOBRE OSTEOPOROSE, DE ACORDO COM O GÊNERO E O TEMPO DE GRADUAÇÃO

\begin{tabular}{lcc}
\hline Gênero & $\mathbf{n}$ & $\%$ \\
\hline Masculino & 201 & 61 \\
\hline Feminino & 129 & 39 \\
\hline Tempo de graduação & & \\
$0-5$ anos & 105 & 31,9 \\
$5-10$ anos & 42 & 12,8 \\
$>10$ anos & 182 & 55,3 \\
\hline
\end{tabular}

A Tabela 2 demonstra que a maioria dos profissionais médicos pesquisados considera que pacientes de meia-idade ( 40 a 66 anos), adultos jovens ( 19 a 40 anos) e idosos (> 66 anos) são os indivíduos em relação aos quais a discussão sobre osteoporose e seu diagnóstico devem sem priorizados.
TABELA 2

NÚMERO DE PROFISSIONAIS MÉDICOS QUE RESPONDERAM AO QUESTIONÁRIO SOBRE OSTEOPOROSE, DE ACORDO COM O GRUPO ETÁRIO O QUAL ACREDITA SER DE RISCO PARA OSTEOPOROSE

\begin{tabular}{lcc}
\hline Grupo etário & $\mathbf{n}$ & $\%$ \\
\hline Crianças (0-8 anos) & 23 & 7 \\
\hline Pré-adolescentes (8-12 anos) & 50 & 15 \\
\hline Adolescentes (12-19 anos) & 92 & 28 \\
Adultos jovens (19-40 anos) & 238 & 72 \\
Meia-idade (40-66 anos) & 287 & 87 \\
Idosos (> 66 anos) & 238 & 72 \\
\hline
\end{tabular}

Os profissionais médicos que participaram do estudo respondendo ao questionário estruturado demonstraram interesse sobre o tema osteoporose. Conforme demonstra a Tabela 3 , cerca de $72 \%$ a $93 \%$ dos participantes disseram estar muito interessados em receber informações concernentes à prevenção e ao tratamento da osteoporose, enquanto $48 \%$ a $60 \%$ deles relataram estar interessados em receber informações sobre pesquisas e serviços destinados ao tema.

TABELA 3

NúMERO DE PROFISSIONAIS MÉDICOS QUE RESPONDERAM AO QUESTIONÁRIO, DE ACORDO COM A NECESSIDADE E O TIPO DE INFORMAÇÃO SOBRE OSTEOPOROSE QUE ACHAVAM CONVENIENTE RECEBER

\begin{tabular}{lcccccc}
\hline Informação & $\begin{array}{c}\text { Gostaria } \\
\text { muito }(\mathbf{n})\end{array}$ & $\%$ & $\begin{array}{c}\text { Gostaria } \\
(\mathbf{n})\end{array}$ & $\%$ & $\begin{array}{c}\text { Não acho } \\
\text { interessante }(\mathbf{n})\end{array}$ & $\%$ \\
\hline Prevenção & 294 & 93 & 13 & 4 & 9 & 3 \\
Tratamento & 228 & 72 & 79 & 25 & 9 & 3 \\
Serviço & 152 & 48 & 108 & 34 & 56 & 19 \\
\hline Pesquisa & 190 & 60 & 102 & 32 & 24 & 9 \\
\hline
\end{tabular}

Setenta e quatro por cento (244) dos participantes trabalhavam em instituições públicas. Pouco mais da metade dos profissionais médicos pesquisados $(55 \%)$ relatou ter fácil acesso à densitometria óssea. Entretanto, apenas 72 médicos $(22 \%)$ relataram ter conhecimento sobre guias de conduta publicados para orientação de conduta no tratamento e prevenção da osteoporose.

A maioria (99\%) dos participantes acredita que é importante ou muito importante prevenir a osteoporose. Da mesma forma, $73 \%$ dos médicos pesquisados acreditam que a osteoporose possa ser prevenida e $63 \%$ deles discutem o 
assunto freqüentemente com os seus pacientes. Por outro lado, como se demonstrou na Tabela 4, mais da metade dos médicos pesquisados não crê que seus pacientes venham a mudar hábitos de vida como forma de prevenção da osteoporose e cerca de $50 \%$ deles não acreditam que seus pacientes venham a aderir ao tratamento da doença em longo prazo. Da mesma forma, apenas 35\% dos médicos pesquisados acreditam que os tratamentos para osteoporose sejam efetivos. Médicos com mais de 10 anos de graduação tenderam a acreditar na eficácia dos tratamentos para osteoporose mais freqüentemente que médicos com menos tempo de graduação, mas essa diferença não foi estatisticamente significativa $(\mathrm{p}=0,08)$.

De forma geral, os médicos pesquisados acreditam que sua formação médica específica para o manejo clínico da osteoporose foi insatisfatória. A Tabela 5 demonstra que $64 \%$ a $79 \%$ dos profissionais médicos pesquisados tiveram pouca ou nenhuma exposição ao tema osteoporose durante a graduação médica ou durante programas de pósgraduação, respectivamente. Por outro lado, a maioria deles $(63 \%)$ acredita que o acesso à informação em osteoporose é fácil, sugerindo que eventuais deficiências na formação médica quanto ao assunto osteoporose possam ser corrigidas. Entretanto, o acesso à informação em osteoporose diferiu entre os médicos pesquisados. Médicos com mais tempo de graduação acharam que o acesso à informação em osteoporose era mais fácil que seus colegas com tempo de graduação inferior a 5 anos $(\mathrm{p}=0,01)$.

Para a maioria dos médicos pesquisados, a história clínica/exame físico e a densitometria óssea são os métodos mais comumente utilizados para o diagnóstico da osteoporose (Tabela 6). Cerca de $72,6 \%$ dos médicos utilizam a anamnese e o exame físico para diagnosticar a osteoporose, enquanto outros $81,8 \%$ fazem uso da densitometria óssea para avaliar fragilidade esquelética na prática clínica. Não houve correlação estatisticamente significativa entre o uso da história clínica/exame físico para o diagnóstico da osteoporose e o tempo de graduação do profissional médico pesquisado. Por outro lado, médicos com 10 anos ou mais de graduação utilizam a densitometria óssea para o diagnóstico da osteoporose mais freqüentemente que seus colegas com menos tempo de graduação $(\mathrm{p}=0,004)$.

TABELA 4

IMPORTÂNCIA DA PREVENÇÃO E DO TRATAMENTO DA OSTEOPOROSE POR PROFISSIONAIS MÉDICOS AFILIADOS À SOCIEDADE Brasileira de Clínica Médica

\begin{tabular}{|c|c|c|c|}
\hline Qual a importância de se prevenir a osteoporose? & $\begin{array}{l}\text { Importante/muito importante } \\
\qquad 326,7(99 \%)\end{array}$ & $\begin{array}{l}\text { Não é importante } \\
0\end{array}$ & $\begin{array}{l}\text { Parcialmente importante } \\
\qquad 3,3(1 \%)\end{array}$ \\
\hline Você acredita que a osteoporose pode ser prevenida? & $\begin{array}{l}\text { Acredito } \\
240,9(73 \%)\end{array}$ & $\begin{array}{l}\text { Acredito parcialmente } \\
\quad 85,8(26 \%)\end{array}$ & $\begin{array}{l}\text { Não acredito } \\
6,6(2 \%)\end{array}$ \\
\hline $\begin{array}{l}\text { Com qual freqüência você discute o assunto osteopo- } \\
\text { rose com seus pacientes? }\end{array}$ & $\begin{array}{l}\text { Freqüentemente } \\
207,9(63 \%)\end{array}$ & $\begin{array}{l}\text { Às vezes } \\
85,8(26 \%)\end{array}$ & $\begin{array}{l}\text { Nunca/raramente } \\
36,3(11 \%)\end{array}$ \\
\hline $\begin{array}{l}\text { Quantos de seus pacientes você acredita que muda- } \\
\text { riam o estilo de vida para prevenir osteoporose? }\end{array}$ & $\begin{array}{l}\text { A maioria } \\
49,5(15 \%)\end{array}$ & $\begin{array}{l}\text { Muito poucos } \\
89,1(27 \%)\end{array}$ & $\begin{array}{l}\text { Nenhum/alguns } \\
191,4(58 \%)\end{array}$ \\
\hline $\begin{array}{l}\text { Quantos de seus pacientes com osteoporose poderiam } \\
\text { aderir ao tratamento em longo prazo? }\end{array}$ & $\begin{array}{c}\text { A maioria } \\
108,9(33 \%)\end{array}$ & $\begin{array}{l}\text { Muito poucos } \\
56,1(17 \%)\end{array}$ & $\begin{array}{l}\text { Nenhum/alguns } \\
165(50 \%)\end{array}$ \\
\hline
\end{tabular}

TABELA 5

FORMAÇÃO MÉDICA ESPECÍFICA SOBRE OSTEOPOROSE E ACESSO À INFORMAÇÃO EM OSTEOPOROSE, DE ACORDO COM profissionais médicos afiliados À Sociedade Brasileira de Clínica Médica

\begin{tabular}{|c|c|c|c|}
\hline Como foi sua exposição ao assunto osteoporose na graduação? & $\begin{array}{c}\text { Mínima/nenhuma } \\
260,7(79 \%)\end{array}$ & $\begin{array}{l}\text { Suficiente } \\
69,3(21 \%)\end{array}$ & $\begin{array}{c}\text { Excessiva } \\
0(0 \%)\end{array}$ \\
\hline Como foi sua exposição ao assunto osteoporose na pós-graduação? & $211,2(64 \%)$ & $112,2(34 \%)$ & $6,6(2 \%)$ \\
\hline Como você qualificaria o acesso à informação sobre osteoporose? & $\begin{array}{c}\text { Muito difícil/difícil } \\
92,4(28 \%)\end{array}$ & $\begin{array}{c}\text { Fácil } \\
207,9(63 \%)\end{array}$ & $\begin{array}{l}\text { Muito fácil } \\
29,7(9 \%)\end{array}$ \\
\hline
\end{tabular}


TABELA 6

MÉTOdos PARA O DIAGNÓSTICO E MONITORAÇÃO DA OSTEOPOROSE DE ACORDO COM PROFISSIONAIS MÉDICOS afiliados à Sociedade Brasileira de Clínica Médica

\begin{tabular}{lll}
\hline & Diagnóstico & Monitoração \\
\hline História/exame físico & $239(72,6 \%)$ & $163(49,5 \%)$ \\
\hline Raio-X de coluna vertebral & & \\
\hline Ultra-sonometria óssea de calcâneo & & \\
\hline Densitometria óssea & $269(81,8 \%)$ & $274(83,8 \%)$ \\
\hline Tomografia computadorizada quantitativa axial & & \\
\hline
\end{tabular}

Um aspecto interessante a destacar é que houve correlação estatisticamente significativa entre tempo de graduação do profissional médico pesquisado e o uso de métodos para a monitoração da osteoporose (Tabela 6). Médicos com tempo de graduação inferior a 5 anos empregavam o uso da história clínica e do exame físico para monitoração da osteoporose mais freqüentemente que seus colegas com 5 anos ou mais de formados $(\mathrm{p}=0,014)$. Da mesma forma, médicos com 10 anos ou mais de graduação utilizavam a densitometria óssea para monitoração da osteoporose mais freqüentemente que seus colegas com menos tempo de graduação. Entretanto, essa diferença não foi estatisticamente significativa $(\mathrm{p}=0,08)$.

\section{DISCUSSÃO}

Nossos resultados indicam que apenas 3\% da amostra total respondeu ao questionário, embora este tenha ficado disponível na web-page da SBCM por 2 anos. Na segunda fase da pesquisa, enviou-se o questionário por e-mail diretamente a todos os médicos sócios da SBCM. Além disso, foi publicado duas vezes no Jornal do Clínico e durante um Congresso de Clínica Médica, que ocorreu nesse período, fez-se um apelo aos participantes para que preenchessem o questionário. $\mathrm{O}$ reduzido número de respondedores limita nossas conclusões, pois, provavelmente, não é representativo da população brasileira de clínicos gerais.

Recentemente, a Sociedade Brasileira de Reumatologia, no intuito de pesquisar, entre reumatologistas do Brasil, as práticas mais comuns em relação a algumas doenças reumatológicas, enviou um questionário para 851 médicos com título de especialistas. Foram devolvidos apenas $21,4 \%$ dos questionários. Os autores sugeriram que o ocorrido poderia refletir uma prática ainda não incorporada à nossa cultura $^{(11)}$. Outros países que realizam pesquisas semelhantes costumam obter $20 \%$ a $50 \%$ de respostas ${ }^{(10,12)}$.
A maioria dos médicos que responderam pertence às Regiões Sul e Sudeste. Tal fato pode ter ocorrido porque há uma grande concentração de médicos de todo o Brasil atuando nessas regiões. Outra possibilidade seria que os médicos dessas regiões têm acesso mais fácil a informações e, conseqüentemente, maior interesse e estímulo para participar de pesquisas.

Mais homens do que mulheres participaram da pesquisa de Taylor et al. ${ }^{(13)}$, utilizando o mesmo questionário na região norte de Londres. Também acreditávamos que as mulheres fossem ter uma participação mais expressiva, visto que a osteoporose é uma doença que tem considerável impacto na saúde feminina. Provavelmente, em nossa amostra, mais homens responderam ao questionário porque na SBCM há mais médicos homens do que mulheres.

A maioria dos médicos da nossa amostra que responderam ao questionário estava formada havia mais de 10 anos. Provavelmente, esses médicos tiveram maior chance de se depararem com pacientes com osteoporose ou foram mais expostos ao tema em congressos e revistas médicas.

Um grande número de clínicos gerais $(72 \%$ a $87 \%)$ que responderam considerou importante discutir o assunto osteoporose com indivíduos a partir dos 19 anos, sugerindo uma preocupação com a prevenção da doença. Resultados semelhantes foram observados por Taylor et $\mathrm{al}^{(13)}$. Embora não tenhamos dados referentes à preocupação dos pacientes em relação a esse tema, nossos resultados sugerem que os clínicos gerais estão procurando ativamente identificar pacientes com risco para osteoporose. Pelo mesmo motivo, demonstraram maior interesse em receber informações sobre prevenção e tratamento da osteoporose do que sobre serviços e pesquisa. Essa necessidade de informação sobre a prevenção e tratamento da osteoporose entre médicos generalistas foi semelhante ao que se observou em um estudo realizado na Inglaterra, no qual 89\% dos médicos pesquisados desejavam essa informação ${ }^{(13)}$.

É interessante notar que embora os médicos considerem importante a prevenção da osteoporose, poucos acreditam que seus pacientes adotariam outro estilo de vida ou teriam maior adesão ao tratamento da doença em longo prazo. Além disso, apenas $35 \%$ dos médicos acreditam que os tratamentos da osteoporose sejam efetivos. Howitt e Armstrong ${ }^{(14)}$ verificaram que a adesão por longos períodos com medicação para doenças crônicas tem sido ruim em diferentes grupos de pacientes. Uma maior adesão desses pacientes ao tratamento poderia ser obtida explicando os riscos e consequiências próprias da doença. Outra forma seria por meio da monitoração da doença e do tratamento 
por meio de marcadores bioquímicos do remodelamento ósseo ou pela densitometria óssea seriada ${ }^{(15,16)}$. Dessa forma, as dificuldades apontadas pelos clínicos gerais de nossa amostra no tratamento da osteoporose, provavelmente, assemelham-se às encontradas no tratamento da hipertensão arterial, diabetes, entre outras. É difícil prevenir e tratar doenças assintomáticas.

A maioria dos médicos $(64 \%)$ considerou que a informação da osteoporose durante a graduação foi mínima. A necessidade para um melhor treinamento dos médicos tem que ser mais difundida. Mudanças no currículo deveriam ser discutidas nas universidades. Outra possibilidade seria a implementação de cursos de educação continuada pelas sociedades médicas envolvidas com o tema. Paradoxalmente, $63 \%$ dos médicos responderam que o acesso à informação sobre osteoporose era fácil, e aqueles com mais de 10 anos de graduação consideraram mais fácil que aqueles com menos tempo de formação. Acreditamos que o acesso à informação seja igual para todos. Possivelmente, os médicos com mais tempo de graduação preocupem-se mais e acabem procurando com mais interesse as informações. Apenas 22\% tinham conhecimento dos guias de conduta. Nossos dados foram semelhantes aos relatados por Jaglal et al. ${ }^{(17)}$ que verificaram que $30 \%$ dos médicos de família em Ontário, no Canadá, tinham conhecimento dos guias de conduta. Taylor et al. ${ }^{(13)}$ relataram que $42 \%$ dos clínicos gerais da região norte de Londres tinham algum conhecimento sobre os guias de conduta.

Pérez-Edo et al. ${ }^{(18)}$, ao realizarem uma pesquisa para determinar a opinião dos clínicos gerais da Espanha em relação ao diagnóstico e tratamento da osteoporose, verificaram que, para fazer o diagnóstico, 96\% dos médicos pediam radiografia, $28 \%$, densitometria óssea, $5,2 \%$, ultrasonometria óssea e $3,1 \%$, tomografia computadorizada quantitativa. A densitometria óssea foi pedida mais freqüentemente pelos médicos que trabalhavam em centros especializados em osteoporose. No estudo EPISER 2000, apoiado pela Sociedade Espanhola de Reumatologia, o diagnóstico de osteoporose na população foi feito por

\section{REFERÊNCIAS}

1. Compston JE, Papapoulos SE, Blanchard F: Report on osteoporosis in the European Community: current status and recommendations for the future. Working Party from European Union Member States. Osteoporos Int 8: 531-4, 1998.

2. Riggs BL, Melton LJ: Involutional osteoporosis. N Engl J Med 314: 1676-86, 1986. radiografias em $82 \%$ dos casos, por testes laboratoriais em $35 \%$ e por métodos de medida da massa óssea em $30 \%$. Surpreendentemente, em nossa amostra, $82 \%$ utilizavam a densitometria óssea para o diagnóstico de osteoporose. Provavelmente, o maior número de densitometrias solicitadas em nosso meio se deva ao fato de que esse exame, além de muito difundido, é reembolsado pela maior parte dos planos de saúde, havendo, inclusive, alguns aparelhos disponíveis na rede pública. Há também um grande interesse dos fabricantes de aparelhos e da indústria farmacêutica em disseminar essa técnica.

Os profissionais com mais de 10 anos de formação pediram mais freqüentemente a densitometria óssea que seus colegas com menor tempo de graduação. Esse dado reforça os achados de que os médicos com mais de 10 anos de formação têm maior exposição a pacientes com osteoporose e, portanto, maior conhecimento.

Nossos dados devem ser interpretados com cautela, pois nossa amostra foi pequena. Além disso, os médicos que responderam ao questionário podem fazer parte de um grupo com maior interesse em osteoporose e, provavelmente, melhor conhecimento e atitudes mais apropriadas em relação à doença. São bem conhecidas as desvantagens de pesquisas baseadas em populações em que as informações não são coletadas sistematicamente. A informação obtida em nosso estudo é essencial para o início do desenvolvimento de estratégias educacionais efetivas para a prevenção e o tratamento de pacientes com osteoporose. Uma maior participação dos médicos nesse tipo de estudo poderia fornecer importantes dados para as tomadas de decisões com os gestores de saúde, tanto das sociedades médicas quanto do sistema público de saúde.

É evidente a necessidade de melhor educação sobre osteoporose e o desenvolvimento de programas específicos para corrigir deficiências no conhecimento dessa doença que afeta milhões de brasileiros.
3. Grupo de Trabajo en Osteoporosis GTO: Estudio de la densidad ósea de la población española. Madrid, Edimsa, 1992.

4. Goeree R, O’ Brien B, Pettitt D, Cuddy L, Feraz M, Adachi J: An assessment of the burden of illness due to osteoporosis in Canadian. J Soc Obstet Gynaecol Can 18:15-24, 1996.

5. Dolan P, Torgerson DJ: The cost of treating osteoporosis fractures in the United Kingdom female population. Osteoporos Int 8: 611-7, 1998. 
6. Keene GS, Parker MJ, Pryor GA: Mortality and morbidity after hip fractures. BMJ 307: 124-50, 1993.

7. Cooper C, Campion G, Melton LJ: Hip fractures in the elderly: a world-wide projection. Osteoporos Int 2: 258-89, 1992.

8. Kiel DP, Felson DT, Aderson JJ, Wilson RWF, Moskowitz MA: Hip fracture and the use of estrogens in postmenopausal women. The Framingham Study. N Engl J Med 317: 1169-74, 1987.

9. Harris ST, Watts NB, Gennant HK, et al.: The effects of risedronate treatment on vertebral and non-vertebral fractures in women with postmenopausal osteoporosis. JAMA 282: 1344-52, 1999.

10. Rowe $\mathrm{R}$. The management of osteoporosis in general practice: results of a national survey. Osteoporosis Rev 7: 1-3, 1999.

11. Medeiros MMC, Ferraz MB, Vilar MJP, et al.: Condutas usuais entre reumatologistas brasileiros: levantamento nacional. Rev Bras Reumatol 45 (2): 82-92, 2006.

12. Cartwright A: Professionals as responders: variations in and effects of response rates to questionnaires. BMJ II: 1419-21, 1978.
13. Taylor JC, Sterkel B, Utley M, et al.: Opinions and experiences in general practice on osteoporosis prevention, diagnosis and management. Osteoporos Int 12: 844-848, 2001.

14. Howitt A, Armstrong D: Implementing evidence based medicine in general practice: audit and qualitative study of antithrombotic treatment for atrial fibrillation. BMJ 318: 1324-7, 1999.

15. Delmas PD, Hardy P, Garnero P, Dain M-P: Monitoring individual response to hormone replacement therapy with bone markers. Bone 26: 553-60, 2000.

16. Eastell R. Treatment of postmenopausal osteoporosis. N Engl J Med 338: 736-46, 1998.

17. Jaglal SB, McIssac WJ, Hawker G, Jaakkimainen L, Cadarette SM, Chan BT: Patterns of use of the bone mineral density test in Ontario, 1992-1998. CMAJ 163: 1139-43, 2000.

18. Pérez-Edo L, Ciria Recasens M, Castelo Branco C, et al.: Management of osteoporosis in general practice: a cross-sectional survey of primary care practitioners in Spain. Osteoporos Int 15: 252-7, 2004. 